\title{
Artificial Rhythms and Cues for Keystroke Dynamics Based Authentication
}

\author{
Sungzoon Cho and Seongseob Hwang \\ Department of Industrial Engineering, Seoul National University, \\ San 56-1, Shillim-dong, Kwanak-gu, Seoul 151-744, Korea \\ \{zoon, hss9414\} @snu.ac.kr \\ http://dmlab.snu.ac.kr
}

\begin{abstract}
Biometrics based user authentication involves collecting user's patterns and then using them to determine if a new pattern is similar enough. The quality of the user's patterns is as important as the quality of the classifier. But, the issue has been ignored in the literature since the popular biometrics are mostly trait based such as finger prints and iris so that its pattern quality depends on the quality of the input device involved. However, the quality of the user's patterns of behavior based biometric such as keystroke dynamics can be improved artificially by increasing the peculiarity of the typing style. In this paper, we propose several ways to improve the quality. But, first we define the quality of patterns in terms of two factors: uniqueness and consistency. Finally, the results of a preliminary experiment are presented that support the utility of the proposed methods.
\end{abstract}

\section{Introduction}

User authentication based on keystroke dynamics has been around for several decades with many papers, patents and products available $[1,2,3,4,5,6$, 7]. There are three steps involved. First, a user registers or enrolls his/her timing vector patterns. Second, a classifier is built using the timing vector patterns. Third, whenever a new timing vector pattern is presented, it is either accepted or rejected based on the classification made by the classifier. Advantages include a low cost, usability and ease for remote access control. A relatively lower accuracy was reported, however, since being a behavior based biometric, keystroke dynamics tends to be less consistent. Recently, however, fairly high accuracies have been achieved through a combination of rather complex models such as neural network, support vector machine, and genetic algorithm $[6,7]$. But, when only a small number of patterns are available, it is difficult to achieve practically acceptable error rates.

Most research efforts related to biometrics based authentication have focused on improving classifier accuracy. In this paper, however, we focus on a different aspect of the problem, i.e. improving the quality of the timing vector patterns. Since keystroke dynamics is a biometric based on user's behavior, patterns can be made "better" by conscious efforts of the user. The quality of the patterns of trait based biometrics such as fingerprint, face, iris and palm prints depends less on the user, and more 
on the input device involved. Thus, improving the quality involves increasing the total cost of the system. The quality of keystroke dynamics patterns can be defined in terms of two factors: uniqueness and consistency. Uniqueness is concerned with how different the impostor's patterns are to those enrolled in the registration stage. Uniqueness tends to depend on the peculiarity of the typing style. Consistency is concerned with how similar the user's patterns are to those enrolled in the registration stage. Consistency depends on the typing skill and the concentration level of the user. A combination of a high consistency and a high uniqueness will lead to a better discriminability or the ability to make better classification of user's patterns and impostors' patterns. In this paper, we propose several ways to increase uniqueness and consistency of keystroke dynamics. For uniqueness, we propose artificially designed rhythms to be used. They include pause, musical rhythm, staccato, legato, and slow tempo. For consistency, we propose timing cues to be used. They include auditory, visual, and audiovisual cues. A preliminary experiment shows that they improve the quality of patterns, thus discriminability.

This paper is structured as follows. The next section presents measures of uniqueness, consistency, and discriminability. Then, several ways to enhance the uniqueness as well as the consistency are proposed along with empirical evidences to support their utility. Finally, a summary and a list of future work are presented.

\section{Uniqueness, Consistency, and Discriminability}

Uniqueness is concerned with how different the valid user's keystroke dynamics is from those of potential impostors. A simple measure of uniqueness can be defined as the average distance of the impostors' typing patterns from the prototype or centroid of the user's typing patterns registered in the enrollment step. Let $\left\{\overrightarrow{x_{i}} \mid i=1, \cdots, N_{x}\right\}$, $\left\{\overrightarrow{y_{j}} \mid j=1, \cdots, N_{y}\right\}$ and $\left\{\overrightarrow{z_{k}} \mid k=1, \cdots, N_{z}\right\}$ denote the valid user's training (enrollment) pattern set, the valid user's test pattern set and the impostors' pattern set, respectively. Given the prototype pattern $\vec{m}=\sum_{i} \overrightarrow{x_{i}} / N_{x}$, Uniqueness is defined as

$$
\text { Uniqueness }=\sum_{k=1}^{N_{z}}\left|\overrightarrow{z_{k}}-\vec{m}\right| / N_{z}-\sum_{i=1}^{N_{x}}\left|\overrightarrow{x_{i}}-\vec{m}\right| / N_{x} .
$$

Consistency is concerned with how similar the valid user's future keystroke dynamics is to the current keystroke dynamics. A simple measure of inconsistency, the opposite concept, can be defined as the average distance of the valid user's own future typing patterns to the prototype or centroid of the user's typing patterns registered in the enrollment step. Inconsistency is defined as

$$
\text { Inconsistency }=\sum_{j=1}^{N_{y}}\left|\overrightarrow{y_{j}}-\vec{m}\right| / N_{y}-\sum_{i=1}^{N_{x}}\left|\overrightarrow{x_{i}}-\vec{m}\right| / N_{x} .
$$


Of course, neither measure can be actually calculated because neither impostor patterns nor future user patterns are available. We can measure these here from the data sets that include user training data, user future test data, and impostor patterns.

As a discriminability measure, we propose to use the difference between the smallest distance from the impostor's pattern to the prototype and the largest distance from the user's future pattern to the prototype. ${ }^{1}$ Now, discriminability is defined as

$$
\text { Discriminability }=\min _{k}\left|\overrightarrow{z_{k}}-\vec{m}\right|-\max _{j}\left|\overrightarrow{y_{j}}-\vec{m}\right| \text {. }
$$

If the former (minimum impostor distance to prototype) is smaller than the latter (maximum user distance to prototype), we obtain a negative discriminability value. Now, one can achieve a perfect discrimination with the use of a proper threshold. We will show that the proposed ways to increase uniqueness and consistency result in a better discrimination.

\section{Strategies to Increase Typing Uniqueness}

In this section, we propose four different ways to increase typing uniqueness. First is inserting any number of pauses. For instance, "pa_ss__word" shows an artificial rhythm containing two pauses of two beats long each. A user types $p$, and a in a natural rhythm, and then inserts a pause of two beats long. Typing $s$ and $s$ in a natural rhythm is followed by another pause of two beats long. Finally, w, o, r, and d are typed in a natural rhythm. Second is typing a password according to a rhythm from certain tune, chant, or rooting. In the experiment, the user employed the rooting rhythm used and popularized by Korean supporters during World Cup 2002 KoreaJapan. The musical rhythm has an advantage. It is easy for a user to remember, thus results in more consistent patterns. A potential disadvantage might be its applicability, particularly to those users with a less rhythmical sense. Third is typing a password with a minimum duration time of each character. So called "staccato" was adopted from a bowing style for string instruments characterized by "being cut short crisply and detached." Staccato typing results in patterns that are very short in duration lengths and that are very uniform in interval lengths. Fourth is the opposite of staccato. In so called "legato" style, one tries to keep each character key down as long as possible, i.e. to maximize duration time of each character. Fifth is typing a password in a slow tempo. Tempo is manifest in the length of interval.

In order to check if these methods are useful, we conducted a simple preliminary test involving one password and one user. Password "password" was chosen. A user typed it in a natural rhythm for 20 times. Then, each of the above mentioned strategies was employed for typing. Two kinds of pauses were tried. For short pauses, "pa_ss_word" explained above was used. For long pauses, "p___assword__" was used which contains two long pauses of three beats and four beats each. Musical rhythm patterns were obtained with the soccer rooting chant mentioned above. Two

\footnotetext{
${ }^{1}$ We have in mind a simple classifier that is based on the distance to the prototype. A typing pattern is accepted if its distance to the prototype is small and rejected if it is large. A proper threshold can be identified.
} 
kinds of staccato were tried: single character staccato and double (two consecutive) character staccato. The double staccato patterns were collected with 'p' and 'a' typed together as fast as possible, followed by the pair of 's' and 's' typed together as fast as possible. The user did not pay any attention to the interval between the two pairs. The pairs of ' $w$ ' and ' $o$ ' and ' $r$ ' and ' $d$ ' were typed in the same manner. The legato patterns were collected as explained above. Also, the slow tempo patterns were obtained as explained above. Note that one password can be converted into many different typing pattern sets, each of which corresponds to a uniqueness enhancing method.

Figure 1 shows the values of inconsistency, uniqueness, and discriminability of seven passwords. Their uniqueness values all increased from at least 200\% (short pauses) to 500\% (slow tempo). Note that the uniqueness values of Long Pauses and Slow Tempo are $1,300 \mathrm{msec}$ and 1,540 msec, respectively so that the corresponding bars in the figure were chopped to fit 1,000. Inconsistency values did not increase much with exceptions of Long Pauses and Slow Tempo. What really matters is the fact that a negative discriminability value of Natural was turned into positive values by employing artificial rhythms. Now all the pattern sets can be perfectly discriminated with a proper threshold.

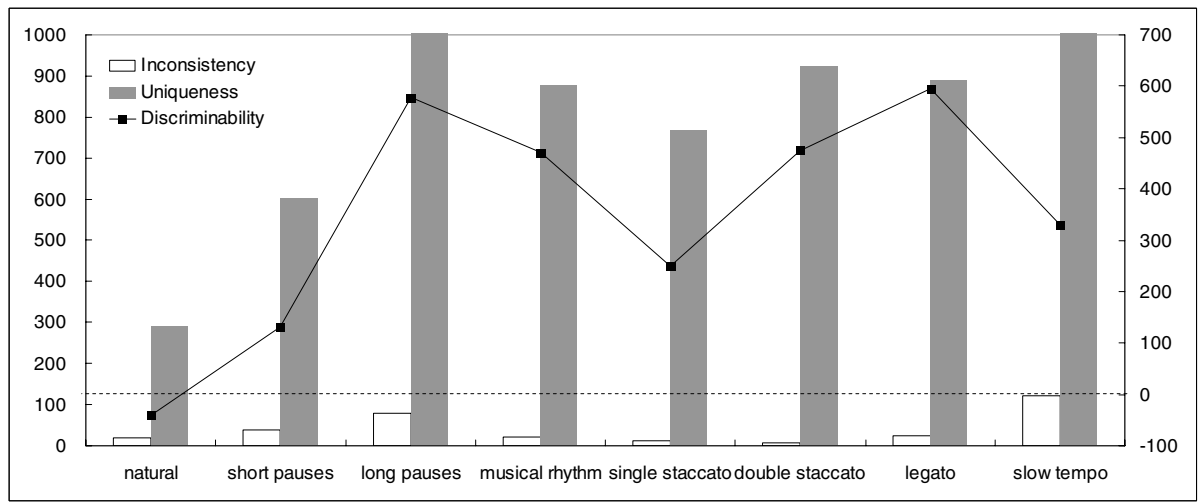

Fig. 1. Uniqueness, Inconsistency (left scale) and Discriminability (right scale) of typing pattern set obtained using natural as well as artificial rhythms. Uniqueness values of Long pauses and Slow tempo are 1,300 and 1,540, respected, but shown here chopped to fit to 1,000.

Figure 2 shows a more detailed picture of what really happened. Each figure contains cumulative distributions of the distances from the training prototype of the user's training patterns (Tr20), the user's test patterns (Test) and the impostors' patterns (Impostor). Discriminability is related to the distance between the solid curve in the middle (Test) and the thick solid curve to the right (Impostor). The farther, the better. When the figure of natural (a) is compared with that of long pauses (b), one thing is clear: the impostor curve is shifted right away from the user test curve. This separation of test patterns and impostor patterns would make a perfect discrimination possible. For (b), its test pattern curve is also pushed right, which was caused by a decrease of consistency in typing. 

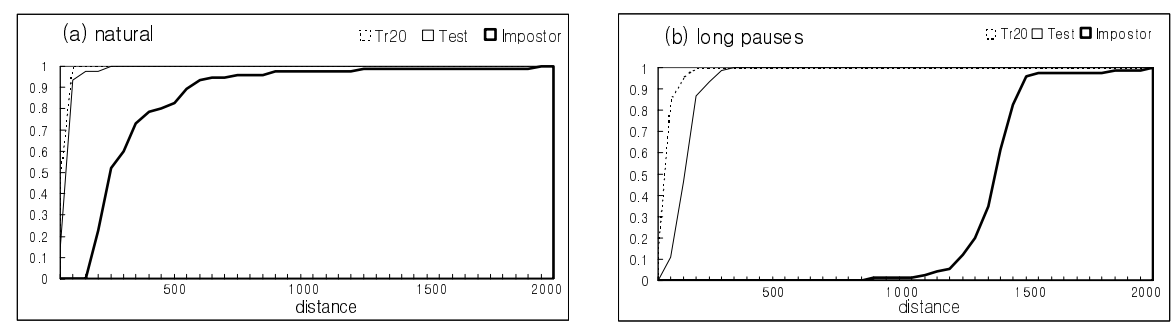

Fig. 2. Cumulative distributions of Distances from Training Prototypes of Tr20 (dotted), Test (solid) and Impostor (thick solid) when the employed method is (a) natural, (b) long pauses

\section{Strategies to Increase Typing Consistency}

In this section, we propose ways to increase typing consistency. First, let us take a look at the slow tempo patterns that had a high inconsistency value in Figure 1. The patterns of slow tempo were collected again in the presence of an auditory cue ticking every 750 msec. The inconsistency value was reduced to 8 from 121. The uniqueness value was slightly reduced to 1436 from 1540 . Thus, the discriminability value was increased to 728 from 330. In short, consistency was improved almost 15 times while discriminability was improved more than twice with a simple auditory cue. See Figure 3 for comparison. User's typing patterns during and after enrollment are quite similar now.

Encouraged by the improvement, we set out to test the effectiveness of various cues with a long pause rhythm of "pass___word___," which contains two long pauses of four beats each. Five different users typed and collected patterns of the long pauses with the following three cues: auditory, visual and audiovisual cue. First, the same speed of 160 per minute ticking sound from a metronome was used for the auditory cue. Second, a video clip of a hammer hitting a nail on a wooden block was presented to the users on the same screen at a speed of 160 per minute. Third, a synchronized combination of both the auditory cue and the visual cue the audiovisual cue was also presented to users as the audiovisual cue. The average uniqueness, inconsistency, and discriminability values over five users for various cues are shown in Figure 4. Note that uniqueness of visual cue is 2,086 so it was chopped at 2,000. Overall, the use of cues clearly decreased inconsistency and increased discriminability.
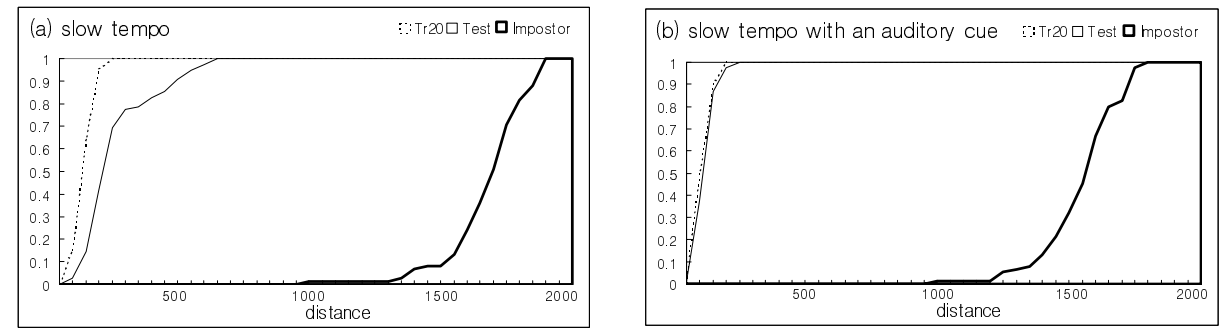

Fig. 3. Cumulative distributions of Distances from Training Prototypes of Tr20 (dotted), Test (solid) and Impostor (thick solid) when the employed method was (a) slow tempo (b) slow tempo with an auditory cue 


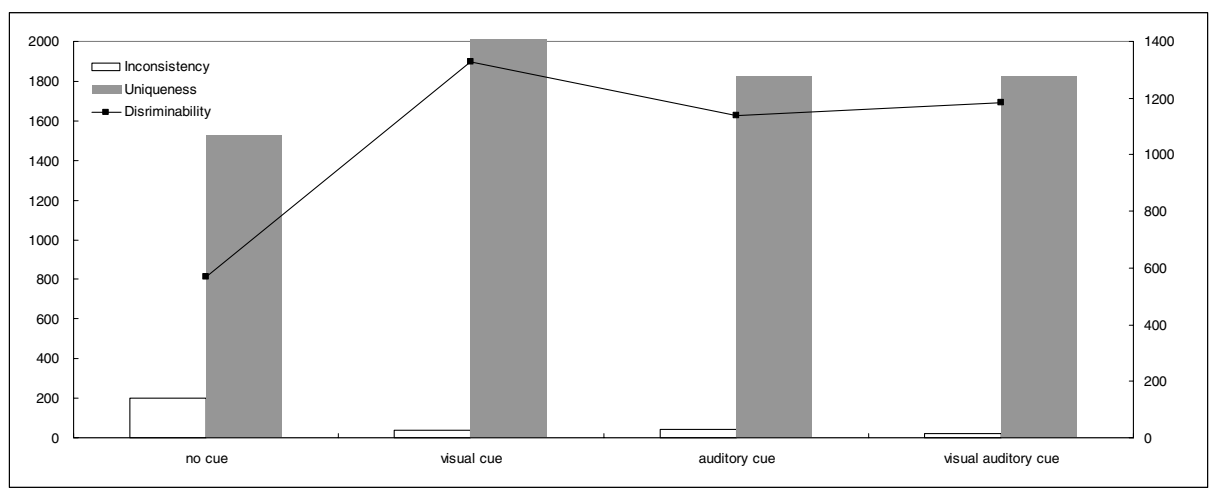

Fig. 4. Uniqueness, Inconsistency (left scale) and Discriminability (right scale) of typing pattern set obtained with various cues used

\section{Conclusion}

This paper proposes several ways to improve consistency and uniqueness in keystroke dynamics. First, for uniqueness improvement, artificial rhythms were suggested. A preliminary test involving one user and five such rhythms was performed. Typing patterns obtained with the proposed artificial rhythms used were found to be significantly more unique than those patterns obtained with a natural rhythm used. The improvement of uniqueness led to better discriminability. Second, for consistency improvement, the use of visual, audio and audiovisual cues was suggested. A preliminary test involving five users and three such cues was performed. Typing patterns obtained with those cues played were found to have decreased inconsistency in all cases. Some cues were found to be more useful to some users.

The ideas and results presented in this work are preliminary. There are many issues to solve and experiments to do. First, much more users and passwords need to be involved in the experiment. Second, artificial rhythms other than those presented here need to be identified. Questions that need to be answered include how easy it is to remember, how long it is to remember, and how consistent typing patterns are in the future. Third, those cues introduced here are the most basic ones. What are the useful cues other than those? Which cues are useful to which users? Is it better to allow a user to choose whatever he/she likes from a menu of various cues? Fourth, it will be interesting to see how the tempo of the cue affects the consistency. Various tempos need to be investigated. Fifth, how this idea of using artificial rhythms and helpful cues translates into situations where an input device other than a keyboard is involved? Examples include cellular phone pads, ATM pads, and some digital door lock pads. Finally, other measures such as ROC should be employed.

\section{Acknowledgements}

This work was supported by grant No. R01-2005-000-103900-0 from the Basic Research Program of the Korea Science and Engineering Foundation. The authors would like to thank Mr. Sung Hoon Park for developing modules that collect keystroke data. 


\section{References}

1. Gaines, R., Lisowski, W., Press, S., Shapiro, N.: Authentication by keystroke timing: some preliminary results. Rand Report R-256-NSF. Rand Corporation (1980)

2. Leggett, J., Williams, G., Usnick, M., Longnecker, M.: Dynamic identity verification via keystroke characteristics. Int. J. Man-Machine Studies. 35 (1991) 859-870

3. Brown, M., Rogers, S.J.: User identification via keystroke characteristics of typed names using neural networks. Int. J. Man-Machine Studies. 39 (1993) 999-1014

4. Obaidat, M., Sadoun, S.: Verification of computer users using keystroke dynamics. IEEE Transactions on Systems, Man and Cybernetics, Part B:P Cybernetics. 27(2) (1997) 261269

5. Cho, S., Han, C., Han, D., Kim, H.: Web-based keystroke dynamics identity verification using neural network. J. Organizational computing and electronic commerce. 10(4) (2000) 295-307

6. Cho, S., Han, D: Apparatus for Authenticating an Individual Based on a Typing Pattern by Using a Neural Network System, Patent No. 6,151,593, Nov. 21, 2000, US Patent and Trademark Office, Washington DC 20231, 2000. (2000)

7. Yu, E., Cho, S.: Keystroke dynamics identity verification - its problems and practical solutions. Computers and Security. 23(5) (2004) 428-440 